\title{
Measurement of noise and its correlation to performance and geometry of small aircraft propellers
}

\author{
Vít Štorch ${ }^{1, a}$, Jiří Nožička1 ${ }^{1}$ Martin Brada ${ }^{1}$, Jiří Gemperle ${ }^{1}$, Jakub Suchý1 \\ ${ }^{1}$ Department of Fluid Dynamics and Thermodynamics, Faculty of Mechanical Engineering, CTU in Prague, Czech Republic
}

\begin{abstract}
A set of small model and UAV propellers is measured both in terms of aerodynamic performance and acoustic noise under static conditions. Apart from obvious correlation of noise to tip speed and propeller diameter the influence of blade pitch, blade pitch distribution, efficiency and shape of the blade is sought. Using the measured performance data a computational model for calculation of aerodynamic noise of propellers will be validated. The range of selected propellers include both propellers designed for nearly static conditions and propellers that are running at highly offdesign conditions, which allows to investigate i.e. the effect of blade stall on both noise level and performance results.
\end{abstract}

\section{Introduction}

The effort in designing aircraft propellers is usually focused to obtaining highest efficiency possible at the design point or throughout a specific design point range. For both fixed and variable pitch propellers, determining the correct operation conditions for a specific application plays also an important role. The propeller can be tailored for maximum efficiency at cruise speed or for a relatively flat efficiency curve that suits aerobatic manoeuvres. According to the required operating range the distribution of geometric properties along the blade are chosen and optimized using an algorithm based on computed airfoil polars and 3D panel method based wake system. Using this technique small fixed pitch model aircraft and UAV propellers have been designed at the Department of Fluid Mechanics and Thermodynamics, CTU in Prague.

Since there is demand on the market for quiet fans and propellers, their noise is one of the priorities of current research. As the first step an experimental measurement is performed on a set of propellers with diameters between 20 and 24 inches in static conditions $\left(v_{\infty}=0\right)$. Although the results cannot be directly used for determining propeller sound power level at operating conditions, their purpose is to validate computation schemes and develop a better understanding of how and if properties like surface roughness, material, chord length distribution, chosen airfoil, pitch angle distribution and overall efficiency affect the sound pressure level, perceived noise and noise spectrum.

The material and geometry of the propeller will affect its aeroelastic behavior. In previous studies it was assumed that the propellers do not deform, in the present work it was decided to perform a simple optical measurement of bending and twisting of the blades due to centrifugal and aerodynamic loads.

\footnotetext{
${ }^{\mathrm{a}}$ Corresponding author: vit.storch $@$ fs.cvut.cz
}

\section{Propeller noise}

There are many different mechanisms of noise generation in turbomachinery and also different ways of their classification $[1,2]$ based on the source type, periodicity and generation mechanism.

The most important frequency in turbomachine acoustics is the blade passing frequency $(B P F)$ of a rotor with $N$ blades rotating with frequency $f$.

$$
B P F=N f
$$

Blade thickness noise is a periodic monopole noise source due to the fluctuating volume of fluid displaced by the blade. It becomes relevant as the tip speed and blade thickness increases. For tip speeds not approaching sonic speed the blade thickness noise is small compared to other sources [2].

Periodic noise due to aerodynamic forces is caused partly by steady pressure distribution relative to the blade which appears as fluctuations at $B P F$ frequency to the observer and its higher harmonics and partly by a nonuniform rotor inflow.

Interaction effects due to the blade passing through stator wakes or its own wake are not important in case of propeller.

Vortex noise belongs to the broadband type of noise as it is caused by vortex shedding at the trailing edge with different periodicity along the blade and blade tip depending on the velocity at each blade span position. A dipole source is produced with strength proportional to the sixth power of section velocity [2].

Turbulence noise is produced in the shear layers, such as the boundary layer, due to small scale turbulence. It has a broadband quadrupole characteristic. 
Propeller is a relatively simple type of open turbomachine which makes it easier to analyse. In studies focusing on propeller noise $[2,3,4]$, the aerodynamic force induced noise is considered as the most important, together with vortex noise that plays an important role in noise reduction efforts [2].

All acoustic measurement results in this paper are presented using no weighting filter, since the main interest lies in the noise generation regardless of frequency sensitivity of human hearing (represented by A filter).

\section{Propeller selection}

Between the samples selected for the measurement were the propellers previously designed at the Department and labelled with D12112 as the manufacturer. For each of these propellers an exact geometry file is available. Commercially available propellers were also selected from several brands. For these propellers, some basic properties, like blade planform and pitch distribution was obtained by processing images. The geometry including airfoil shape can be also scanned using a manual 3D scanner developed in-house, which is however a very lengthy process and has not been used in the current work. The list of selected propellers can be found in table 1 .

Table 1. Selected propellers for measurement

\begin{tabular}{|c|c|c|c|}
\hline Make & Designation & $\begin{array}{c}\text { Measured } \\
\text { pitch } \\
\text { [inch/rev }]\end{array}$ & Surface finish \\
\hline D12112 & $20 \times 13 \mathrm{E} 3$ & 16.8 & Raw CF \\
\hline D12112 & $20 \times 13 \mathrm{E} 4$ & 15.9 & Raw CF \\
\hline D12112 & $22 \times 10 \mathrm{E}$ & 14.1 & Raw CF \\
\hline PT & $20 \times 12 \mathrm{WE}$ & 13.5 & Coated CF \\
\hline PT & $20 \times 13 \mathrm{E}$ & 16.2 & Coated CF \\
\hline PT & $21 \times 13 \mathrm{E}$ & 15.1 & Coated CF \\
\hline PT & $22 \times 8 \mathrm{HP}$ & 11.8 & Coated CF \\
\hline PT & $22 \times 10 \mathrm{E}$ I. & 10.8 & Coated CF \\
\hline PT & $22 \times 10 \mathrm{E}$ II. & 11.3 & Coated CF \\
\hline APC & $22 \times 10 \mathrm{E}$ I. & 12.3 & $\begin{array}{c}\text { Fiber plastic } \\
\text { composite }\end{array}$ \\
\hline Mejzlik & $20,5 \times 12 \mathrm{WEL}$ & 15.1 & Coated CF \\
\hline Mejzlik & $24 \times 8$ EVO2 & 12.0 & Coated CF \\
\hline RS & $21 \times 14 \mathrm{EL}$ & 15.4 & Coated CF \\
\hline
\end{tabular}

The main parameters of a propeller is the diameter and pitch. Since the distribution of pitch is usually not constant along the blade, an effective pitch is considered as being the pitch of the blade at $70 \%$ of radius. However, our experience has shown that manufacturers of small propellers tend to indicate lower values, perhaps to gain an advantage over competition in thrust at a specific pitch. Some past geometry measurements of the authors have shown up to $3 \mathrm{inch} /$ revolution difference in measured vs. indicated pitch so it was decided to process the images to obtain the pitch distribution and calculate a representative value of pitch taken as the average value between $40 \%$ and $90 \%$ of blade span.

The propellers measured are between $20 \div 24$ inches $(508 \div 609.6 \mathrm{~mm})$ in diameter with pitch in range of $8 \div 14$ inches per revolution. They have three different surface finishes, with coated carbon fiber being the finest and raw carbon fiber the coarsest. Letter $\mathrm{E}$ in the designation stands for electrical as the recommended type of motor. $\mathrm{W}$ is used by some manufacturers to indicate wider blades, if they make two versions of the same diameter and pitch. Also the number after $\mathrm{E}$ in case of D12112 propellers is the prototype version, meaning that E3 and E4 propellers have slightly different geometry.

To get an idea about the precision and reliability of the measurement, one propeller is represented by a pair of identical samples denoted by I. and II. (identical except for manufacturing imperfections).

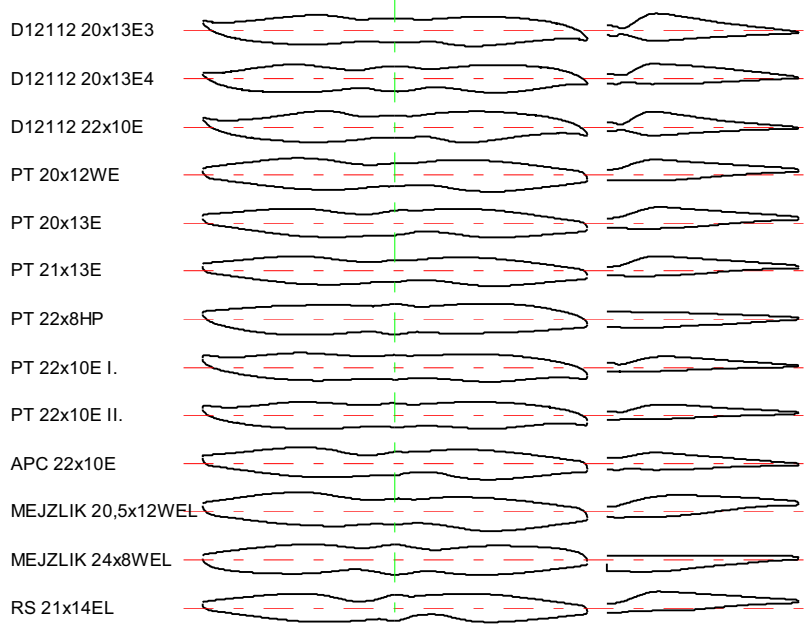

Figure 1. Planforms and side views of propellers (not rotating) obtained by processing images.

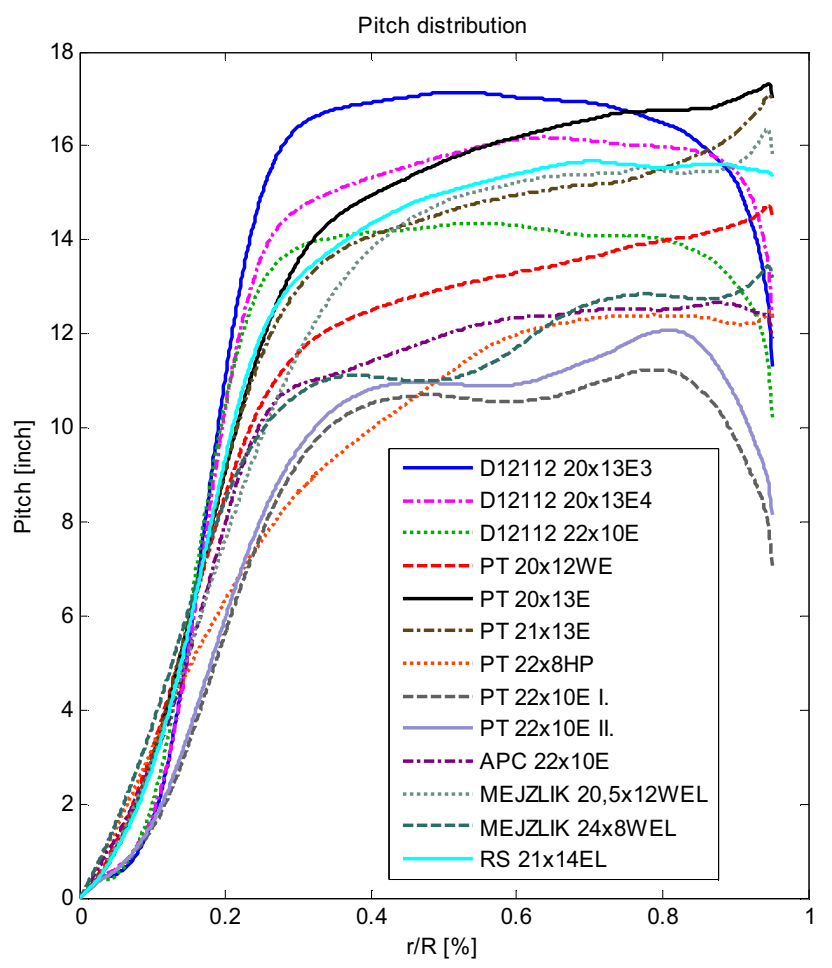

Figure 2. Pitch distribution based on image processing

Last but not least it must be noted that none of the selected propellers was designed to work under static conditions and the results provided cannot be used for 
judging the quality of each propeller. On the other hand, noise of propellers working in more or less off-design conditions promise more variable results.

\section{Measurement}

\subsection{Experimental stand and data acquisition}

The available wind tunnel for propeller measurement in dynamic conditions could not be used for acoustic measurement, due to the noise of the tunnel fan. It was decided to build a simple stand for static measurement. The stand consists of a welded hexagonal cage with motor mount.

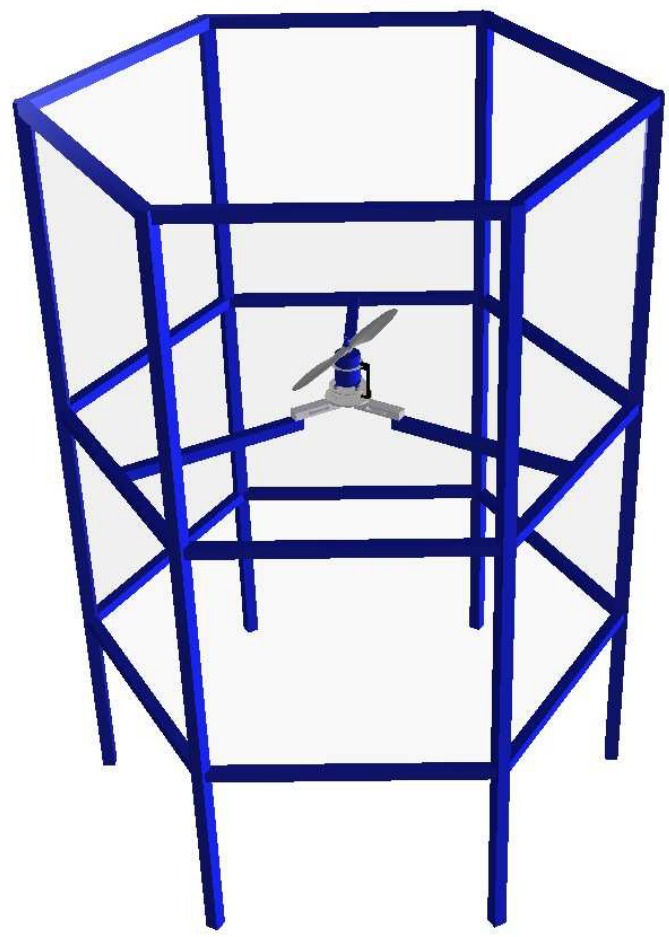

Figure 3. The measurement stand consists of a welded hexagonal frame with protective net on the sides.

The motor mount contains in total 4 load cells for measurement of thrust force and torque. An optical gate together with perforated ring mounted on the motor's casing provides the measurement of rotational speed and also provides synchronization signal for the flash. All these devices are connected to an in-house designed electronic circuit with ADCs and the data read into MATLAB. For the purpose of studying aeroelastic behaviour of the propeller a DSLR camera is mounted on a tripod and positioned to capture the blade from the side. The exposure time was set at $1 / 10 \mathrm{~s}$, which allowed for several blade passes during one exposure. After opening the shutter, the camera sends a signal to the flash to fire. This signal is delayed until the optic gate confirms correct position of the blades and the flash can fire. The logical circuit also limits the flashes to only one during one exposure. Canon EX430II was used as the flash and gives approximately $0.05 \mathrm{~ms}$ long light impulse at the lowest power settings. For the highest measured rotational speed of $105 \mathrm{~Hz}$ the blade rotates aproximately $2^{\circ}$ during the flash duration.

The acoustic quantities such as the overall sound pressure level and FFT spectrum are measured using NTI XL2 analyzer connected to a NTI M2211 omnidirectional, free-field microphone. The microphone is placed $550 \mathrm{~mm}$ from the axis of rotation and $100 \mathrm{~mm}$ below the propeller disc plane.

\subsection{Experiment procedure}

Due to the large set of propellers, it was decided to measure the performance of each propeller at 4 different rpms equivalent to $40,60,80$ and $100 \%$ of electronic throttle. Because the measurement is done under static conditions the results should be similar (when expressed in dimensionless coefficients) with the exception of small deviations due to different Reynolds numbers.

At each measurement point the propeller was rotating at a fixed speed and the values of rpm, thrust force and torque were recorded simultaneously for 5 seconds at a $1000 \mathrm{~Hz}$ sampling rate and averaged to a single value. Two images were captured by the camera and the FFT spectra were recorded by the sound analyzer in the ranges: $7 \mathrm{~Hz} \div 215 \mathrm{~Hz}, 58 \mathrm{~Hz} \div 1.7 \mathrm{kHz}$ and $484 \div 20 \mathrm{kHz}$

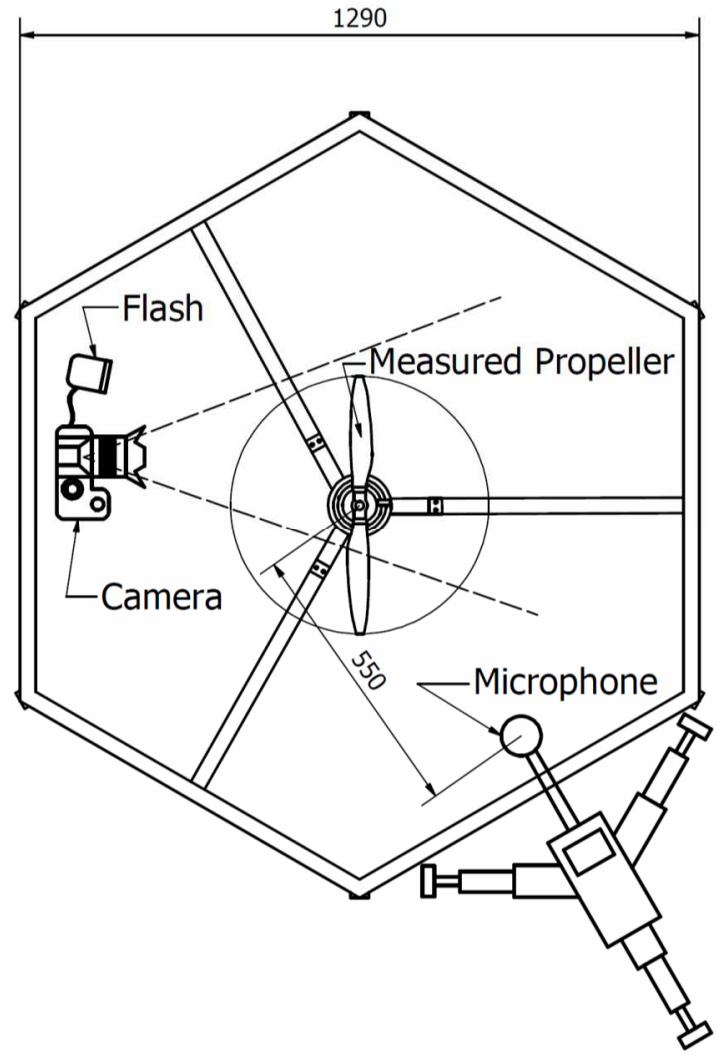

Figure 4. Measurement scheme. The microphone is placed on a tripod to avoid vibrations from the main frame.

\section{Aeroelasticity measurement results}

After processing the images several curves normalized to the tip radius $\mathrm{R}$ were obtained for each propeller and each measurement point. Based on the side view, the actual 
thickness $\mathrm{C}_{\mathrm{T}}=\mathrm{C}_{\mathrm{T}}(\mathrm{r} / \mathrm{R})$ and midline $\mathrm{C}_{\mathrm{M}}=\mathrm{C}_{\mathrm{M}}(\mathrm{r} / \mathrm{R})$ curves were calculated. Midline was defined as the curve averaging the leading edge and trailing edge curves. By subtracting the midline of the propeller at $0 \mathrm{rpm}$ from each midline of rotating propeller, bending curves were obtained. By combining the actual blade thickness and the blade width distribution $\mathrm{C}_{\mathrm{W}}=\mathrm{C}_{\mathrm{W}}(\mathrm{r} / \mathrm{R})$ obtained from the planform photos, a formula for twist $\mathrm{C}_{\Phi}$ distribution at i-th measured rotational velocity could have been derived:

$$
C_{\Phi}(i)=\operatorname{atan}\left(\frac{C_{T(i)}}{C_{w}}\right)-\operatorname{atan}\left(\frac{C_{T(0 r p m)}}{C_{w}}\right)
$$

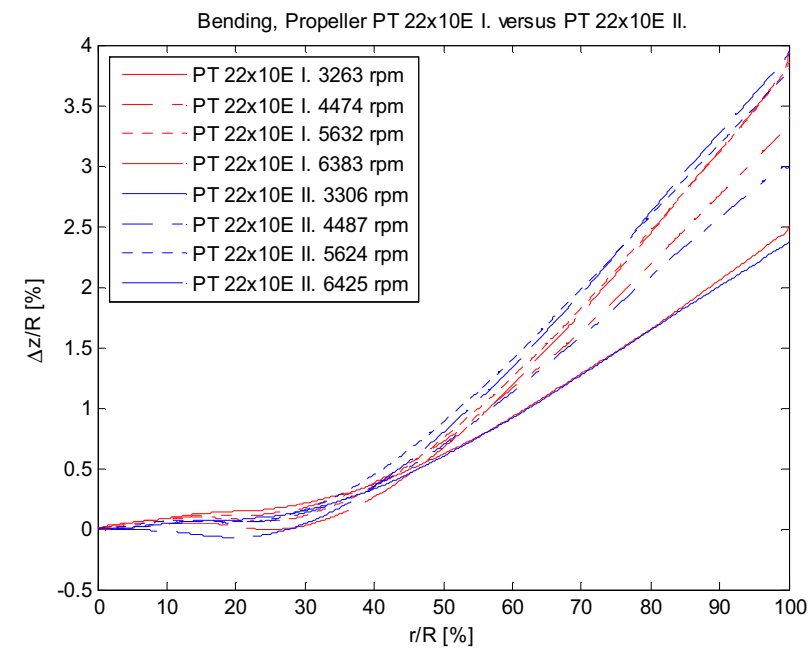

Figure 5. Typical shape of bending curves demonstrated on the two propellers PT $22 \times 10$ E sample I. and II.

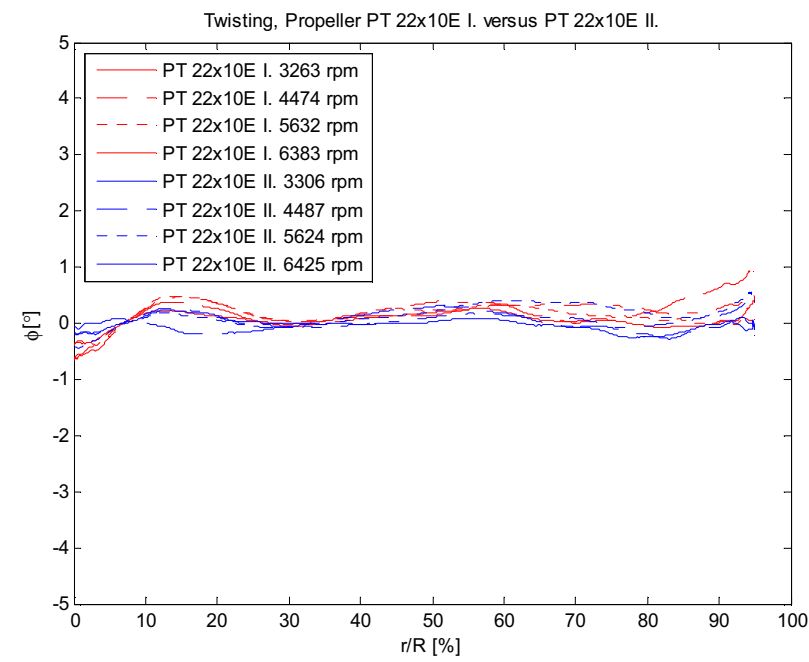

Figure 6. Typical results for twisting curves demonstrated on the two propellers PT 22x10E sample I. and II.

After running the algorithm on all samples, it was shown, that all propellers bend quite noticeably forward. By comparing the results of two samples of the same propeller model (figure 5) the measurement method produces very similar bending curves. It is interesting to note, that after a certain rotational speed the bending displacement seem to stop increasing, which is probably due to centrifugal force preventing further bending.

The angle of twist due to aerodynamic loading on the other hand stays within 0.5 degrees from zero for most blades, which seem to be also the accuracy of the method, as can be seen in figure 6 .

The main purpose of this part of the measurement - to get information about changes in geometry due to aerodynamic loading was accomplished. The twisting angle falls below measurable values, so it can be assumed that the blades do not twist. Bending, on the other hand, is well measurable and depending on the material and construction of the blade the blade tip will bend forward by $0.5 \div 4 \%$ of the tip radius. The results were stored for current and future numerical calculations, however some test calculations have confirmed the assumption that bending of the propeller has much smaller influence on the performance of propeller then its twisting.

\section{Noise measurement results}

The measurement could not be performed in an isolated anechoic chamber so an attention had to be payed to the microphone position and background noise levels. An example of measured sound spectrum is in figure 6 . The difference in overall SPL (sound pressure level) between propeller noise and background is in this case $53 \mathrm{~dB}$ which is more than sufficient to neglect the background noise.

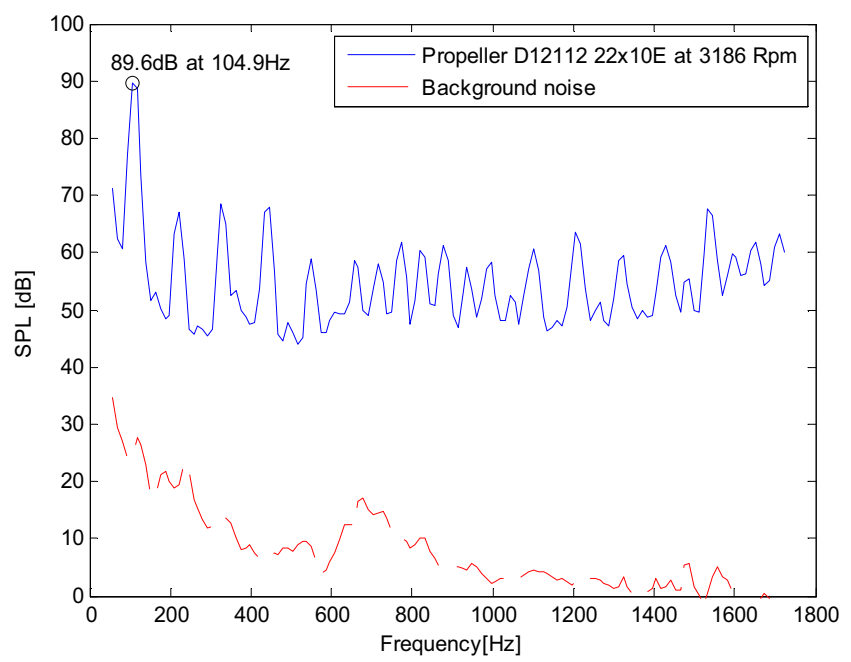

Figure 7. An example of the sound spectrum for a low rpm case of the $22 \times 10 \mathrm{E}$ propeller with labelled peak value together with background noise.

The noise spectrum in figure 7 is representative of all measured propellers, the highest peak is always at the $B P F$, which is for a two bladed propeller twice the frequency of rotation. The following peaks are at higher harmonic frequencies. The obtained overall SPL of each propeller was plotted against rpm, tip speed, shaft power and thrust power $P_{T}$ calculated from the thrust by the propulsion equation:

$$
P_{T}=\sqrt{\frac{\left(F_{T}\right)^{3}}{2 \rho A}}
$$

Where $F_{T}$ is the thrust and $A$ the propeller disc area.

The resulting curves are presented in figures 8-11. The $S P L$ grows with rpm and tip speed in a linear manner, whereas the SPL is roughly proportional to the cubic root of shaft and thrust power, which can be expected from 
well-known relation between fan power and rpm. Figure 8 shows the expected fact that at constant revolutions, propellers of higher diameter and pitch tend to produce higher SPL and when constant tip speed is used for comparison, the higher pitch (and therefore greater section loading) produces higher noise levels.

More interesting are the plots of $S P L$ vs. shaft power and thrust power. The data in figure 10 form a relatively narrow band, which means that the shaft power is the parameter that influences noise levels the most. On the other hand the thrust power curves are quite scattered, which confirms that the aerodynamic efficiency plays a crucial role also in noise reduction efforts.

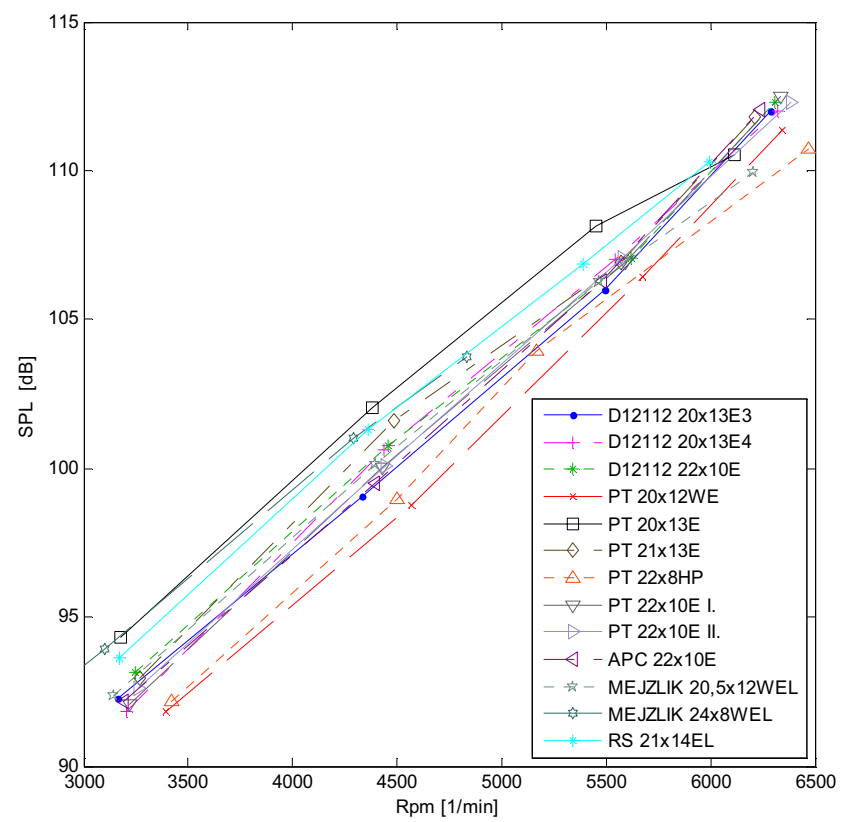

Figure 8. Sound pressure levels plotted against rpm

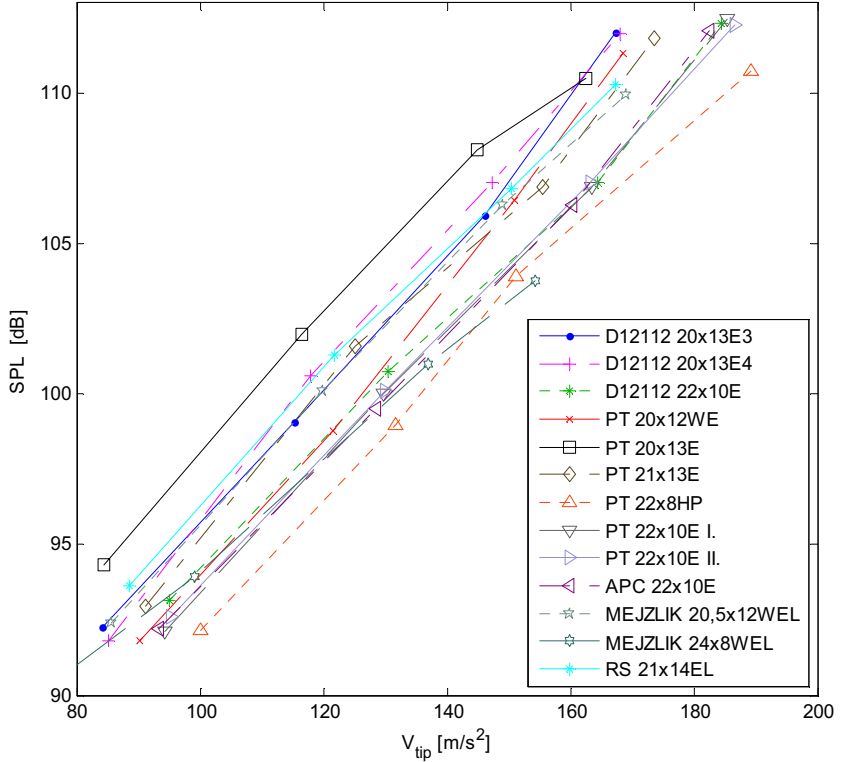

Figure 9. Sound pressure levels plotted against the tip speed.

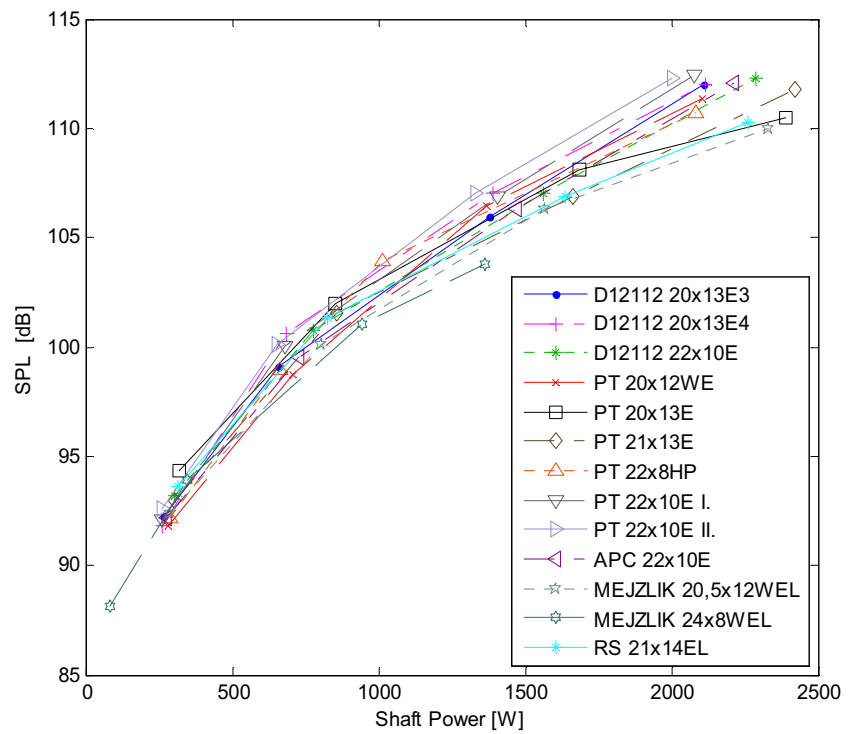

Figure 10. Sound pressure levels plotted against the shaft power

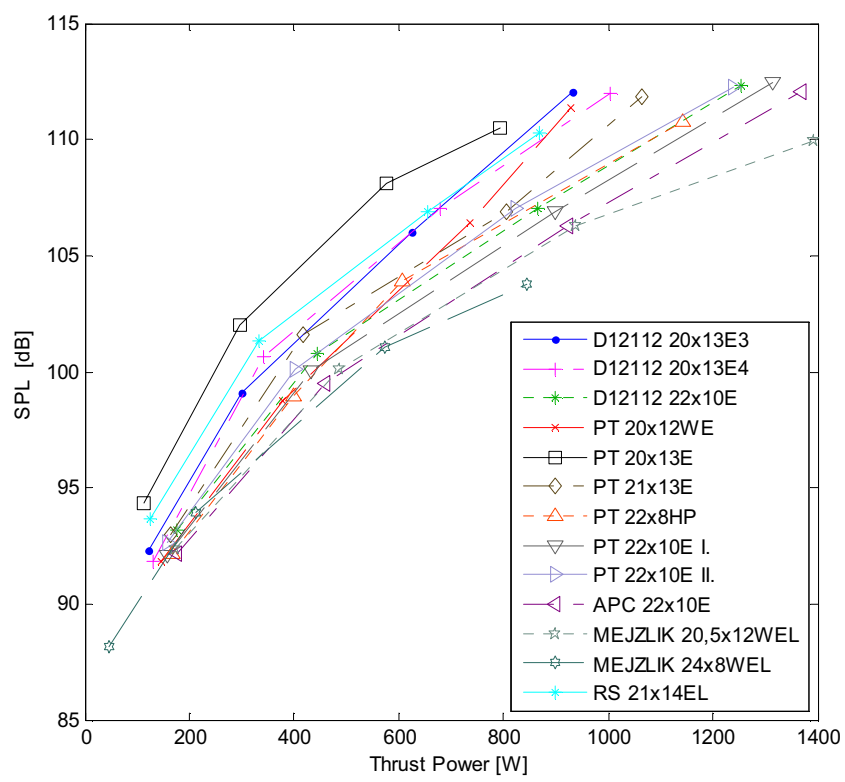

Figure 11. Sound pressure levels plotted against the thrust power

In order to be able to compare the measured propellers more efficiently values of $S P L$ were interpolated from the graphs at constant chosen parameters and visualised using bar graphs (figures $12 \div 15$ ). Interesting comparison of propellers in figure 14 shows that when constant shaft power is provided, more efficient propellers (note: efficiency at static conditions) tend to produce more noise. This is not true in all cases, but the tendency is noticeable. This can be explained by a hypothesis that the noise due to dissipation in a less efficient propeller (stalled and large vorticity flow) does not reach the same level of noise as a propeller with efficient aerodynamic loading (large pressure gradients of well streamlined sections ) when both propellers are powered by the same shaft power.

The SPL at constant thrust power (figure 15) shows, that at constant thrust power, the noise is almost inversely proportional to the efficiency. This can be expected from the close tie between $S P L$ and shaft power. 


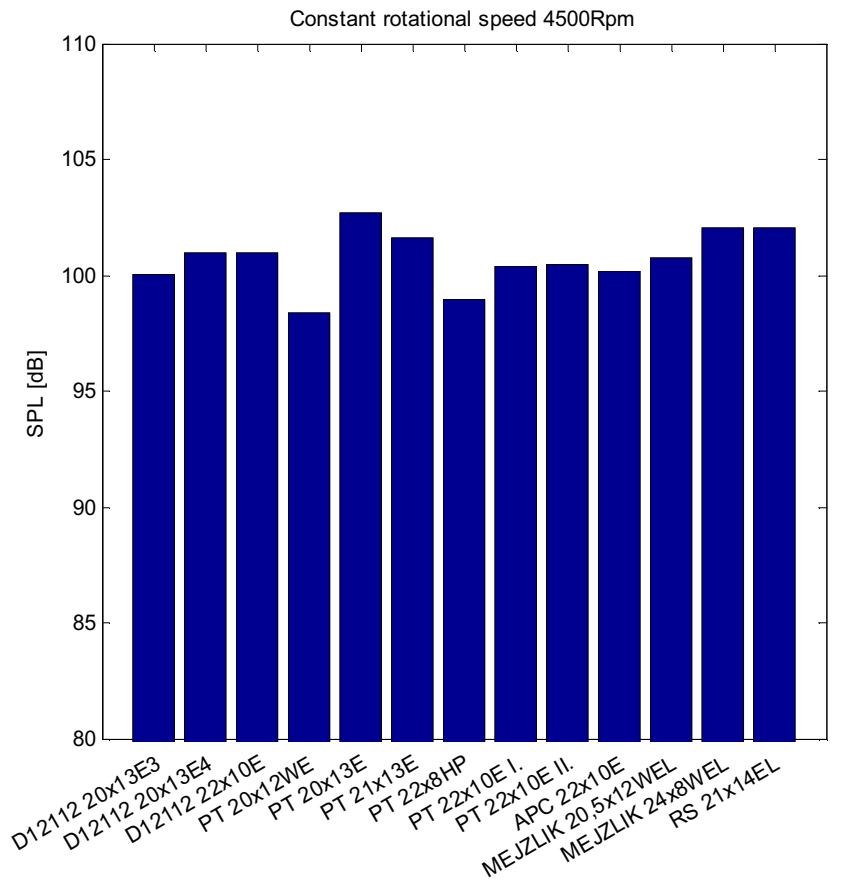

Figure 12. Sound pressure levels at $4500 \mathrm{rpm}$

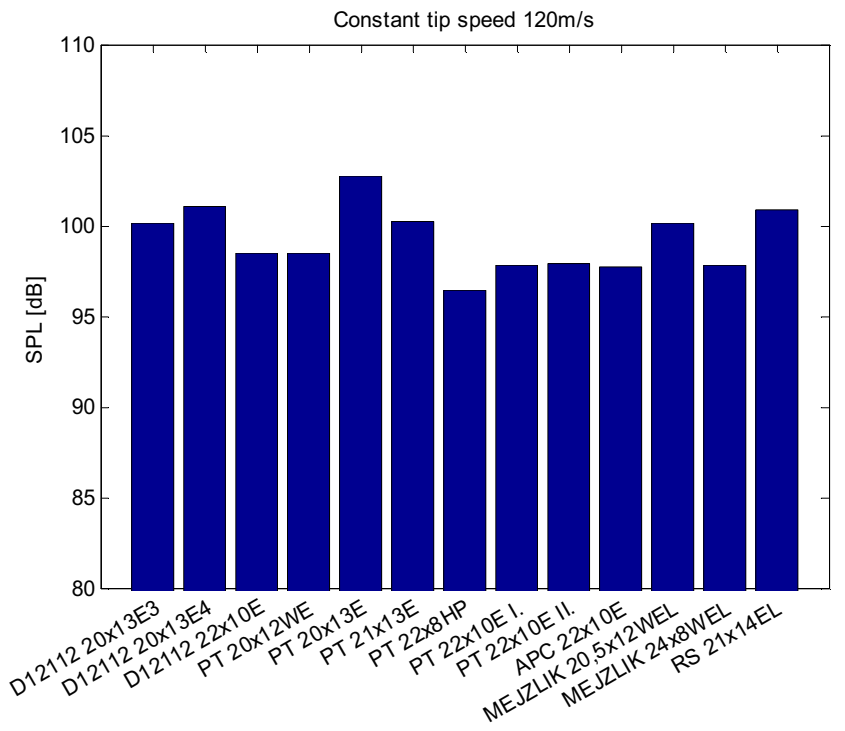

Figure 13. Sound pressure levels at $120 \mathrm{~m} / \mathrm{s}$ tip speed

\section{Conclusion}

The presented work focuses on three main areas regarding small aircraft propellers - geometry, aerodynamics and acoustics. By inspecting the blade geometry it was confirmed, that the average pitch angle does not correspond to the designation of the propellers, and the pitch and chord distributions for future calculations were obtained. Image acquisition during blade rotation revealed little or no blade twisting but significant bending of the blades.

The aerodynamic performance measurement was done using proven setup for obtaining thrust, torque and rpm data. For each measurement point, the noise spectrum was recorded. The presented results will be used for the current development of computational model of propeller performance and acoustics.

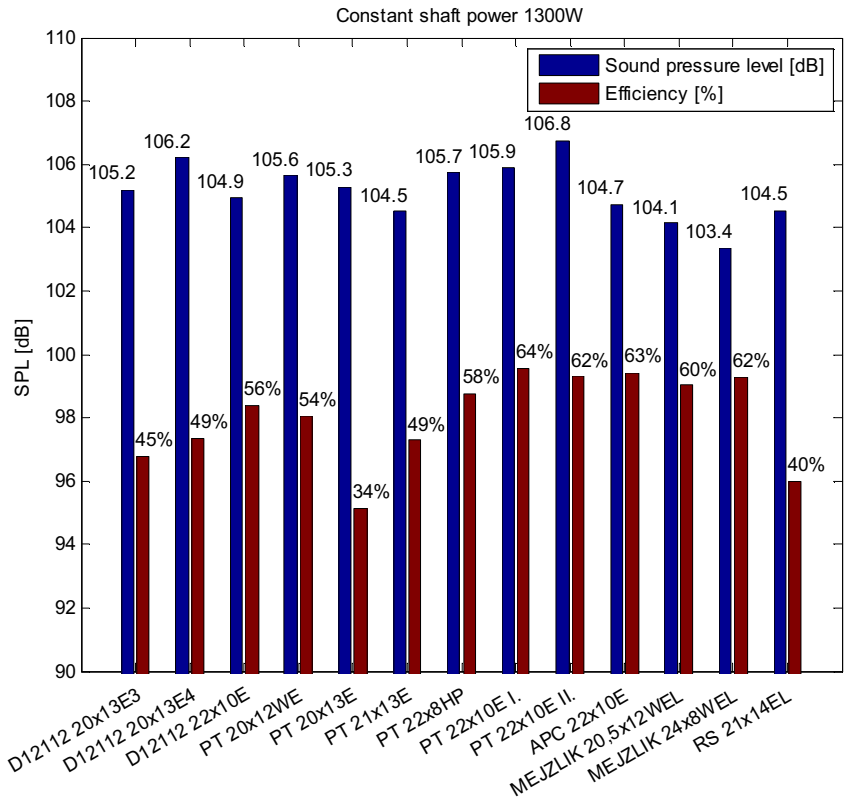

Figure 14. Sound pressure levels and efficiency at $1300 \mathrm{~W}$ shaft power

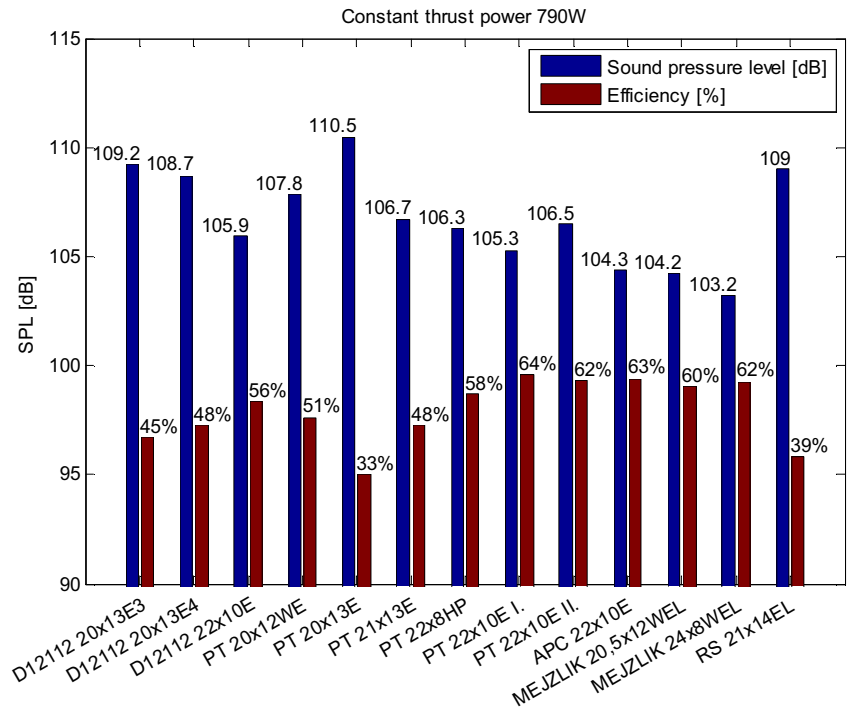

Figure 15. Sound pressure levels and efficiency at 790W thrust power

\section{Acknowledgement}

This work was supported by the Grant Agency of the Czech Technical University in Prague, grant No. SGS 15/065/OHK2/1T/12

\section{References}

1. W. Cory, Fans and Ventilation, (Elsevier Science, Amsterdam Boston, 2005)

2. J. Marte, D. Kurtz, A Review of Aerodynamic Noise From Propellers, Rotors and Lift Fans, (Jet Propulsion Laboratory, Pasadena, 1970)

3. M. Carley, J. Sound Vib. 233 (2000) 255-277

4. G. Sinibaldi, L. Marino, Appl. Acoust. 74 (2013) 7988 